\title{
A case report of an Infant erysipelas on a impetigo
}

\section{Abdellah Dah Cherif, Mohammed Chaouche, Younes Barbach, Sara Elloudi, Hanane Baybay, Fatima Zahra Mernissi}

Department Dermatology, University Hospital Hassan II, Fez, Morocco

Corresponding author: Dr. Abdellah Dah Cherif, E-mail: abdellahdahcherif@gmail.com

\begin{abstract}
Erysipelas is a relatively common infection of the upper dermis and superficial lymphatics. It affects mainly adults, between 40 and 80 years of age, especially immunocompromised ones, but some cases were reported also in newborns and infants. Its pathogenesis mostly involves a disruption of the skin barrier allowing the infective agent, mainly group a b-hemolytic streptococcus, to enter. It most commonly involves the lower extremities or the face, presenting as a welldemarcated, tender, tense, deeply erythematous and indurated plaque. These lesions may spread more rapidly in infants than in older patients and seems to undergo rapid exacerbations at times until the administration of treatment. We report an unusual pediatric case of a leg erysipelas on a impetigo in a 24-month-old infant with good result.
\end{abstract}

Key words: Erysipelas; Dermo-Hypodermitis; Infant; Skin infection 


\title{
Un impétigobulleuxcompliquant un érysipèle chez un nourrisson
}

\section{Abdellah Dah Cherif, Mohammed Chaouche, Younes Barbach, Sara Elloudi, Hanane Baybay, Fatima Zahra Mernissi}

Department Dermatology, University Hospital Hassan II, Fez, Morocco

Corresponding author: Dr. Abdellah Dah Cherif, E-mail: abdellahdahcherif@gmail.com

\begin{abstract}
RÉSUMÉ
Lérysipèleestune dermo-hypodermitebactérienneaiguë non nécrosante,iltouche avec prédilection les adultes et les sujetsâgésmaisilpeut affecter aussi les enfants, affectant le plus souvent les membresinférieurs, qui se manifeste par un érythèmecirconscrit, accompagné d'un œè̀me local et d'un étatfébrile. Les facteursfavorisantsasurvenuesont les portesd'entrée locales, les oedèmes de toutes causes et des facteurssystémiques (diabète, immunosuppression). Le diagnosticestavanttoutclinique. La complication la plus fréquenteest la récidive. Les autrescomplicationsincluentnot ammentl'abcédassion, la fasciite et les bactériémies. Ilestrare de pouvoiridentifier le germe de l'érysipèle. La majorité des infections (85\%) estliéeaustreptocoquebêta-hémolytique du groupe A. Le traitement de l'érysipèle consiste en une antibiothérapieassociée à un repos de la régionconcernée, nousrapportonsdanscetarticle un cas d' érysipèle sur un impétigo chez un nourrissonavec une bonneévolutionsoustraitement.
\end{abstract}

Mots clés: Erésipèle; Dermo-hypodermite; Nourrisson; Infection cutanée

\section{INTRODUCTION}

Lérysipèleestune dermo-hypodermiteaigue, due le plus souvent au streptocoque bétahémolytique du groupe A. Il touche avec prédilection les adultes et les sujetsâgésmais Il peut affecter aussi les enfants. Le diagnostic est clinique. On rapporte un cas d' un érysipèle dont la porte d'entraitétait un impétigo bulleux chez un nourrisson.

\section{CASE REPORT}

Il s' agit du nourrisson D.M, de 22 mois, sans antécédents pathologiques particuliers, qui présente depuis 15 jours des lésions de contenu liquidien sur les 2 membres inferieurs, sans aucun traitement, hospitalisé pour une grosse jambe rouge aigue douloureuse évoluant depuis 2 jours avant son admission.

À l'examen clinique on trouve, un enfant conscient, stable sur le plan hémodynamique et respiratoire, fébrile à $39^{\circ} \mathrm{C}$, l' examen dermatologique trouve un placard érythémateux et odémateux, chaud et douloureux de la jambe gauche débordant vers la cuisses, surmonté par des bulles tendues et des croutes mélicériques, avec un lymphoedeme important, sans purpura, nicrépitation, ni trouble de sensibilité (Fig. 1) et une adénopathie inguinale homolatérale.

Le bilanbiologique: hyperleucocytose de20920/mm3 à prédominanc epolynucléaires neutrophiles de 16025 avec CRP de 240mg/l, et une échographie des parties molles objectivant une infiltration des parties molles diffuse sans individualisation de collection visible.

Lenfant mis sous Amoxicilline-acideclavulanique a dose de $50 \mathrm{mg} / \mathrm{kg} / \mathrm{j}$, avec une bonne évolution clinique et biologique après $48 \mathrm{~h}$ du traitement(Fig. 2) la durée du traitementétait de 14 jours, avec une protéinurie de 24 h était normale après 3 semaine de son hospitalisation.

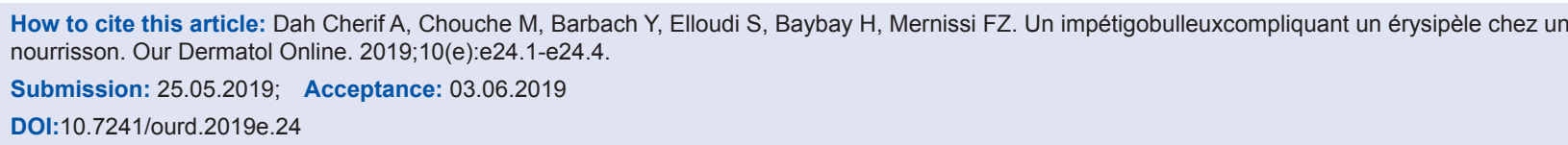




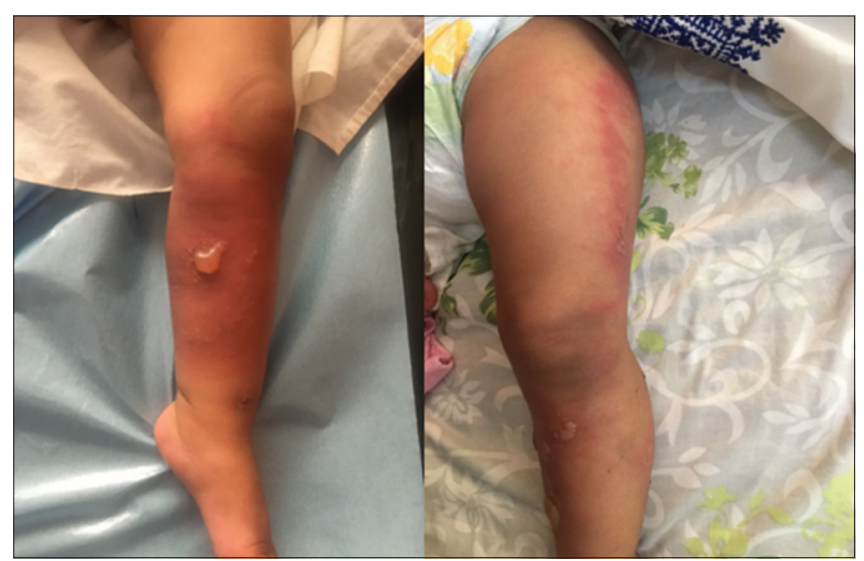

Figure 1: Un placardérythémateux et œdémateux, chaud et douloureux de la jambe gauchedébordantvers la cuisses, surmonté par des bulles tendues.

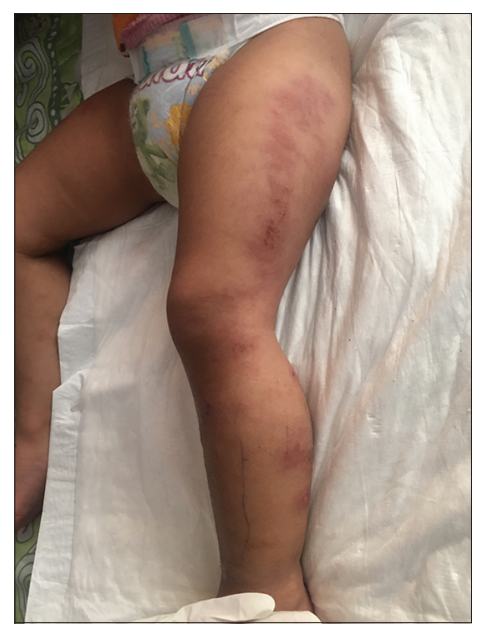

Figure 2: Aspect de la jambe après $48 \mathrm{~h}$ du traitement.

\section{DISCUSSION}

L' érysipèle est une infection cutanée aiguë, donnant un tableau clinique de dermo-hypodermite non nécrosante, due à un streptocoque de groupe $\mathrm{A}$ ou SPA), autres groupes: $\mathrm{B}, \mathrm{C}$ ou $\mathrm{G}+++$, Streptococcus pneumoniae et streptocoques de groupe $\mathrm{D}+/$-, Staphylocoques, BGN (Pseudomonas aeruginosa, Acinetobacter calcoaceticus, entérobactéries.), Haemophilus influenza, associations microbiennes streptocoques et staphylocoques [1]. Cette infection cutanée suite a une porte d'entrée qui doit être systématiquement recherchée et sera retrouvée dans deux tiers des cas, sous forme d'intertrigo, de mycose interdigitale, de plaies, de piqûre ou d'un ulcère. Les facteurs favorisants locaux comme les œdèmes de toutes causes (lymphodème et insuffisance veineuse), ou les dermatoses chroniques (par exemple, psoriasis), ainsi que les facteurs favorisants systémiques comme le diabète l'immunosuppression, l'alcoolisme, le tabagisme...). doivent être documentés [2]., Cliniquement il se manifeste brutalement par une fièvre de 38,5 à $39^{\circ} \mathrm{C}$ voire $40{ }^{\circ} \mathrm{C}$. accompagnée généralement de frissons; un placard rouge, chaud, et douloureux, siégeant dans plus de $85 \%$ des cas, aux membres inférieurs (jambes, pieds, chevilles, cuisses). Elle peut aussi être siégeant au niveau du visage, aux membres supérieurs, tronc, et parfois même aux organes génitaux [3]. Une adénopathie homolatérale inflammatoire satellite voire une trainée de lymphangite. Le diagnostic de l'érysipèle est clinique, mais un bilan biologique faite de NFS et CRP est nécessaire pour confirmé le diagnostic [4]. Les Complications peut être locales (abcès, nécrose superficielle, complications infectieuses ostéoarticulaires, thrombophlébite, abcès rétropharyngé, médiastinite et pleuropéricardite), ou générales: bactériémies (septicémies), toxidermies (pénicilline), décompensation de tares, mortalité tardive, les récurrences: récidives (avec un germe différent), rechutes (avec le même germe), endocardite et glomérulonéphrite aiguë [4].

La prise en charge: il existe plusieurs critères d' hospitalisations, critères liées aux modalités du traitement la pénicilline $G$ par voie intraveineuse et le repos au lit, critères liées aux terrain: œedèmes locorégionaux, lymphoedème, oedème chronique, l'obésité, le diabète, alcoolisme, les maladies cardiovasculaires, la toxicomanie, âge avancé, ou très précoce comme le cas de notre malade. Lantibiothérapie systémique doit être avant tout antistreptococcique: $\beta$-lactamines en monothérapie: Pénicilline $G$ injectableest le traitement de référence mais de moins en moinsutilisée, Pénicilline A orale: amoxicilline, Amoxicilline + acide clavulanique $(50 \mathrm{mg} / \mathrm{kg}$ en 3 prisesquotidiennes) en cas de doute sur la possibilité d'autre bactérie en cause (Staphylococcusaureus), comme le cas de notre patient; Macrolides: Synergistines: pristinamycine, en cas d'allergie à la pénicilline; Clindamycine: (effetsindésirables digestifs) [5]. Les mesures associées: repos aulitavecsurélévation des membres inférieurs, traitement anticoagulantpréventif à associer seulement s'il y a un risque patent de maladie thromboembolique associée. Antalgiques en cas de douleur et un traitement adapté de la porte d'entrée. AINS systémiques sont formellement déconseillés (le risque évolutif vers la nécrose pourrait être plus important?). Prophylaxie secondaire: traitementd'uneported'entrée persistante (antifongique topique encas d'intertrig omycosique) [2], prise en charge des facteurs favorisants 
(insuffisance veinolymphatique, obésité, etc.). En cas de récidives multiples, une antibiothérapie au long cours possible [6].

\section{CONCLUSION}

Lérysipèleestuneentitécliniquesouventbénigne, qui touchetous les âges, moinsfréquemment les enfants.La pénicilline $\mathrm{G}$ est la molécule de référence. L'évolutionestsouventbénigne, cependant, des complications graves peuventémailler le coursévolutif de ces infections.

\section{Consent}

The examination of the patient was conducted according to the Declaration of Helsinki principles.

\section{REFERENCES}

1. Sočan K, Sočan M. Trends in the epidemiology of erysipelas in Slovenia. Acta Dermatovenerol Alp Pannonica Adriat. 2018;27:1-4.

2. Binnick AN, Klein RB, Baughman RD. Recurrent erysipelas caused by group B streptococcus organisms. Arch Dermatol. 1980;116:798-9.

3. Noblesse I, Carvalho P, Young P, Hellot MF, Levesque H. Facteurs de gravité des érysipèles de jambe. Ann Dermatol Venereol. 1999;126(suppl 2):S65.

4. Jorup-Ronstrom C. Epidemiological, bacteriological and complicating features of erysipelas. Scand J Infect Dis. 1986;18:519-24.

5. Chartier C, Grosshans E. Erysipelas. Int J Dermatol. 1980;29:459-67.

6. Dalal A, Eskin-Schwartz M, Mimouni D, Ray S, Days W, Hodak E, et al. Interventions for the prevention of recurrent erysipelas and cellulitis. Cochrane Database Syst Rev. 2017;6:CD009758.

Copyright by Abdellah Dah Cherif, et al. This is an open access article distributed under the terms of the Creative Commons Attribution License, which permits unrestricted use, distribution, and reproduction in any medium, provided the original author and source are credited.

Source of Support: Nil, Conflict of Interest: None declared. 